\title{
Louky Bersianik
}

\section{L'espace encombré de la signature}
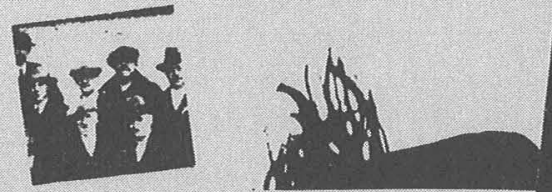

En tant que lectrice, la première chose du texte que je lis, c'est sa signature. C'est là qu'est le premier contexte, le premier référent. Et jamais, autant que dans ce contexte qui le colore et lui donne tout son sens, le texte n'est-il appelé à vibrer, à dire quelque chose en plus. Ou à se taire, parce qu'il y a des signatures qui tiennent en respect d'éventuelles lectrices (ou lecteurs éventuels). Forme pleine avant même que n'advienne la matière du texte, la signature serait donc le lieu privilégié de l'énonciation. Même si tout ce qui la précède peut sembler objectif, distancié, voire indifférent, la signature garantit au texte sa subjectivité, lui confere même le sceau de la responsabilité. La signature est un signe : c'est la marque du sujet écrivant. Aux textes anonymes importants, on prête volontiers une signature. Dieu est l'auteur de la Bible!

Toutes les oeuvres signées ne portent pas nécessairement le nom de leurs vraies auteures (ceci est valable aussi pour les vrais auteurs). Parfois, au moment de signer, on s'aperçoit que la place est déjà prise. Quelqu'un de plus malin, de plus rapide, a rempli l'espace alloué, l'a encombré de son nom, lui faisant porter le poids de ses erreurs. On le reconnaît à ce signe particulier : c'est également lui qui signe les enfants humains, oeuvres féminines s'il en est, là où la mère se perd, là où la femme toujours déjà n'existe pas. Fille garçon, son enfant est regardé comme un dérivé du père, il est déclaré tel, le nom qu'on lui donne est sa première dérive, son premier détournement. (Si c'est une fille, elle est mise entre parenthèses ou sous la 
rigidité de la barre oblique, ou demeure invisible tout simplement). N'ayant jamais pris racine en l'homme, l'enfant se trouve annexé/e à lui dès sa sortie du ventre, comme à un radical étranger sa terminaison nerveuse, sa désinence naturelle, par un nouveau cordon ombilical qui ne sera jamais coupé et qui lui fera reproduire tout au long de son existence les formes du paradigme paternel. Flexion radicale, modification de l'être à partir d'un thème, d'un élément emprunté, inflexion vocalique, changement du timbre normal de la voix sous l'influence d'un suffixe patronymique ajouté automatiquement à la tête de chaque enfant, signature du patriarcat imprimée sur toute chair humaine dès sa venue en ce monde. Dans la signature de l'auteure, je vois la figure impertinente et impérieuse du patriarcat, s'incrustant aussi en filigrane sur la page même qui le dénonce. Mais l'auteure est forcée de continuer à l'inscrire car c'est son identité nominale. Qui agit comme un titre nominatif i.e. qui porte le nom du propriétaire. En endossant le système patriarcal dont elle cautionne ainsi la légitimité, cette fausse inscription fait dévier la circulation des objets textuels (car tels qu'en d'autres qu'eux-mêmes ils sont changés par elle), et fait ainsi obstacle à leur libre usage.

Le nom désigne la catégorie à laquelle appartiennent les êtres et il sert aussi à les distinguer. Le prénom indique presque toujours le sexe d'une personne humaine, le patronyme indique la filiation fondée sur la patrilinéarité. Le nom de famille est le signe visible de l'organisation du monde patriarcal. Ce n'est qu'après coup qu'il réfere à tel ou tel père en particulier. Ce qui est gênant dans le patronyme, ce n'est pas la présence du père (ou du père du mari), c'est l'absence de la mère. 
Le nom de famille ignore la filiation à la mère comme si celle-ci n'était un membre de la famille qu'au même titre que les enfants qui en sont issus. Cette absence joue dans toutes les circonstances, même lorsque les auteures prennent le nom de leur mère car ce nom, ainsi que le nom de la mère de cette mère (et ainsi de suite ad nauseam), c'est encore et toujours le nom du père. Il est donc juste de dire que la femme n'a pas de nom. Elle n'a qu'un prénom... dans le meilleur des cas. C'est ce qui apparaît clairement quand on examine les généalogies des familles, l'histoire des femmes célèbres et les signatures d'auteures.

Le pseudonyme n'est pas une solution de continuité. car, non seulement ne fait-il pas apparaître la matri-. linéarité, mais il est toujours organisé de telle façon que sa deuxième partie, en occupant la place du nom de famille, est perçue comme le patronyme, et donc fait référence au père, au chef de famille. D'ailleurs, quand il est connu comme tel, le pseudonyme est reconnu comme étant un faux nom, une fausse identité (pseudo est un élément du grec pseudès qui veut dire «menteur»). Il n'est qu'un paraphe, qu'une signature abrégée, et n'offre que de fausses «initiales». Le pseudonyme ment sur ses origines, mais, comme tel, il ne tente de masquer que le père (et non la mère puisque, de toute façon, elle n'apparaît jamais dans le «vrai nom»). Le pseudonyme est un faux, un nom d'emprunt : il nie la famille patriarcale mais il en emprunte le nom puisqu'il se forge un "nom de famille».

Dans un autre sens, on pourrait dire que toutes les femmes ont un pseudonyme puisque toutes portent un nom d'emprunt. Mais on pourrait le dire aussi de tous 
les hommes. Non pas parce que les unes et les autres portent le nom du père, mais parce que toutes et tous ne portent que ce nom-là, ce qui est une aberration généalogique. La signature du père, dont est constitué le nom patronymique, est une légende parce qu'elle fait croire que les êtres humains ne sont issus que du mâle. C'est de la pure mythologie : c'est Athéna qui proclame ne pas avoir eu de mère puisqu'elle est sortie de la tête de Zeus, c'est Adam qui s'enorgueillit de ce que la femme n'est qu'une côte tirée de son thorax, c'est l'homme moderne qui appelle "porteuse» la mère biologique de l'enfant (comme la porteuse de pain ou la porteuse de nom...). Le mot légende signifie littéralement : ce qui doit être lu. Toute signature de textes est, quelque part, suspecte, sujette à caution, parce que légendaire, i.e. fabuleuse, imaginaire, mythique, qui n'est vraie qu'à moitié. En tant que légende, elle a donc un rapport direct avec la lecture et doit être lue et relue comme telle, i.e. comme un objet douteux. Je parle d'une lecture attentive, «créatrice», qui permet d'en «négocier le sens» et d'en réécrire le texte. Celui-ci semble précéder la signature sur la page mais en fait il la suit et de très loin. C'est cette distanciation, entre ce que les signataires s'imaginent être en signant leurs textes et ces textes eux-mêmes, qui doit apparaître clairement dans une lectureréécriture créatrice.

Comme c'est le nom du père qui fait trace dans la signature, il est évident que cette lecture-réécriture ne sera pas la même quand elle sera exercée par des femmes sur des textes signés par des hommes que dans la situation contraire. À date, presque tous mes textes ont été des relectures-réécritures - sous forme d'interrogation 
et de théorie-fiction - de différents chapitres du bagage culturel patriarcal qu'on appelle communément les «sciences humaines» d'une part : la religion, l'histoire, la mythologie, la philosophie, la psychologie, l'anthropologie, la sociologie, la linguistique (le langage : langue et parole), etc. et les dictionnaires qui en rendent compte; ainsi que ce qui en a découlé : la littérature et les oeuvres d'art d'autre part. Non seulement fautil interroger les textes qui sont tous situés dans un contexte historique de société patriarcale, mais aussi les subjectivités qui les ont produits dans le même contexte. Toutes les écrivaines qui ont une conscience féministe intense ne peuvent faire l'économie de ce travail de lecture créatrice des textes patriarcaux, car c'est seulement de cette façon qu'elles pourront traverser sans encombre - et faire traverser de même leurs lectrices et lecteurs - l'espace de leur propre signature. 